\title{
Diagnostic yield and safety of ultrasound- assisted biopsies in superior vena cava syndrome
}

\author{
C.F.N. Koegelenberg*, C.T. Bolliger*, D. Plekker*, C.A. Wright”, K. Brundyn\#, \\ M. Louw" ${ }^{\#}$, P. Schubert ${ }^{\#}$, M.M. van den Heuvel* and A.H. Diacon*,
}

ABSTRACT: The yield and safety of ultrasound (US)-assisted transthoracic fine needle aspirations (TTFNA) and cutting needle biopsies (CNB) in the setting of superior vena cava (SVC) syndrome are unknown. The aims of the present prospective study were to asses the diagnostic yield and safety of US-assisted TTFNA and CNB in SVC syndrome with an associated mass lesion abutting the chest wall.

Over a 3-yr period, the present authors screened 59 patients with SVC syndrome, and enrolled 25 patients who had an associated mass lesion that extended to the chest wall. US-assisted TTFNA with rapid on-site evaluation (ROSE) was performed in all cases. CNBs were performed where a provisional diagnosis of bronchogenic carcinoma could not be established, and in $57.1 \%$ of patients with bronchogenic carcinoma (limited due to safety constraints).

ROSE of US-assisted TTFNA confirmed diagnostically useful material in 24 patients, and cytological diagnoses were ultimately made in all of these cases (diagnostic yield 96\%). USassisted CNB had a diagnostic yield of $\mathbf{8 7 . 5 \%}$. Minor haemorrhage occurred in one out of 25 TTFNA and three out of $16 \mathrm{CNB}$. Neither procedure resulted in major haemorrhage nor pneumothoraces.

US-assisted TTFNA and CNB have a high diagnostic yield and are safe in the setting of SVC syndrome with an associated mass lesion abutting the chest wall.

KEYWORDS: Bronchogenic carcinoma, cutting needle biopsy, superior vena cava syndrome, transthoracic fine needle aspiration, ultrasound

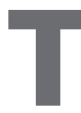
he superior vena cava (SVC) syndrome is a clinical entity caused by obstruction of the superior vena cava by infiltration, compression or thrombosis [1]. Intrathoracic neoplasms, mainly bronchogenic carcinomas and lymphomas, are by far the most common causes [1-3]. An SVC syndrome secondary to an intrathoracic neoplasm is generally considered an oncological emergency that requires an expedient diagnostic evaluation and initiation of treatment [1, 4]. Bronchoscopy and associated procedures have a reported diagnostic yield of $>70 \%$ in this setting, whereas mediastinoscopy or mediastinotomy have a diagnostic yield of $>90 \%[5,6]$. The latter procedures are associated with a high anaesthetic risk and a relatively high complication rate, and major haemorrhage is seen in a significant percentage of cases due to high venous pressure $[5,6]$. Moreover, associated brain oedema may complicate mediastinoscopy or mediastinotomy, as patients may experience significant discomfort in the supine position [1].
Thoracic ultrasound (US) is a well-established diagnostic aid to the clinician [7-10]. The major advantages of this modality include its dynamic properties, low cost, lack of radiation, mobility and short examination time [7-12]. US of the chest is increasingly being used to guide interventional procedures, such as thoracentesis, and biopsies of tumours of the chest wall, pleura or peripheral lung [7]. Intrathoracic mass lesions abutting or invading the chest wall are visible at US [7-9]. Recent studies on US-guided transthoracic fine needle aspirations (TTFNA) utilising rapid on-site evaluation (ROSE) and US-assisted cutting needle biopsies (CNB) of mass lesions involving or abutting the pleura have shown that these procedures are not only safe but also have a high diagnostic sensitivity [13, 14].

There is, however, a paucity of data on the diagnostic yield and safety of US-assisted TTFNA with ROSE and CNB in the setting of SVC syndrome caused by mass lesions that either

\section{AFFILIATIONS}

*Division of Pulmonology, Dept of Medicine,

-Division of Medical Physiology, Dept of Biomedical Sciences, University of Stellenbosch and Tygerberg Academic Hospital, and "Division of Anatomical Pathology, Dept of Pathology, University of Stellenbosch and National Health Laboratory Services, Cape Town, South Africa.

CORRESPONDENCE

C.F.N. Koegelenberg Division of Pulmonology Dept of Medicine University of Stellenbosch P0 Box 19063

Tygerberg

7505

Cape Town

South Africa

Fax: 27219333591

E-mail: coeniefn@sun.ac.za

Received:

August 202008

Accepted after revision:

December 172008

STATEMENT OF INTEREST

None declared.

European Respiratory Journal Print ISSN 0903-1936 Online ISSN 1399-3003 
abut or invade the chest wall. The aims of the present prospective study were to assess the feasibility, diagnostic yield and safety of these investigations in the setting of SVC syndrome and, specifically, in the hands of nonradiologists.

\section{METHODS}

\section{Study population}

All adult patients ( $\geqslant 18$ yrs of age) with a clinical diagnosis of SVC syndrome referred to the Division of Pulmonology of Tygerberg Academic Hospital, Cape Town, South Africa, were potential candidates for the present 3-yr prospective analytical observational study. Tygerberg Academic Hospital is a 1,200bed university hospital. It is one of two referral centres and renders a tertiary service to a population of $\sim 1.5$ million people. The Committee for Human Research of the University of Stellenbosch, Cape Town, South Africa, ethically approved the study. Written informed consent was obtained from all subjects on enrolment and also prior to any invasive procedures.

A contrasted computed tomography (CT) scan of the chest and upper abdomen was performed on all patients, according to standard operating procedure at the Tygerberg Academic Hospital. Studies routinely included the supraclavicular fossae and lesions were classified on radiological appearance as to the most likely origin: "mediastinal" (lesions predominantly located in the anterior mediastinum with extension to the pleura), "pulmonary" (lesion with centre in the lung, acute angle to the pleura), "pleural" (pleural based, blunt angle to the lung) or "chest wall" (lesions centred in the chest wall with pleural involvement). All CT scans were scored by an independent radiologist with regards to the maximum length of the interface between the particular lesion and the thoracic wall (in the longest plane). The depth of lesions relative to the pleural surface and the presence of chest wall invasion were also documented.

The initial evaluation of all patients included, where applicable, fine needle aspiration (FNA) of palpable and/or radiologically detectable supraclavicular lymph nodes or diagnostic pleural aspirations. Only cases with confirmed partial or complete SVC obstruction that remained undiagnosed following these investigations and where an intrathoracic mass lesion abutted the chest wall to an extent that it made a FNA feasible were enrolled onto the present prospective observational study.

\section{Thoracic US}

A consultant respiratory physician or a senior registrar under supervision performed the sonography (Toshiba Just Vision 200 SSA-320A; Toshiba Medical Systems Corporation, Tochigiken, Japan). All procedures were performed in a bronchoscopy suite without the support of a specialist radiologist. Although the preferred patient position for the procedure was supine, with a parasternal approach, the clinician could employ a more lateral or apical approach if this was deemed feasible and safe. The operator reviewed the thoracic CT scan prior to performing the transthoracic US. The interface between the intrathoracic mass lesion and chest wall was then identified by means of a standard $3.75-\mathrm{MHz}$ sector probe. An interface of $\geqslant 1 \mathrm{~cm}^{2}$ visible throughout inspiration and expiration in an area not covered by bony elements was considered the minimal requirement for an US-assisted procedure. Procedures were performed "freehand" (i.e. not under direct US guidance). The intended site was marked, and the direction, the depth of interest and the safety range for the procedure documented. The patient was instructed not to change position in order to prevent a positional shift of the area of interest relative to the skin mark. Care was taken to avoid the major intrathoracic blood vessels, and the intercostal and mammarian arteries, as well as any major collateral veins that may have formed secondary to the SVC obstruction.

\section{TTFNA}

Under sterile technique and local anaesthesia with lignocaine $1 \%$, aspirations were performed with a $22-\mathrm{G}$ spinal needle (40 or $90 \mathrm{~mm}$; Becton Dickinson, Madrid, Spain) connected to a $10-\mathrm{mL}$ syringe. Aspirates from at least four slightly different directions and depths were directly expressed onto slides, smeared and submitted for ROSE using Diff-Quik (Rapidiff; Clinical Sciences Diagnostics, Southdale, South Africa) and rapid Papanicolaou staining methods.

\section{ROSE of cytology specimens}

The cytopathologist present in theatre was experienced in ROSE and was asked to comment on the presence or absence of diagnostically useful material obtained during each individual pass. Material that was considered not diagnostically useful included blood, necrotic tissue or the total absence of cellular material. Where diagnostically useful material was present, the cytopathologist was asked to make a provisional diagnosis of malignant or nonmalignant pathology. Where malignant cells were present, the pathologist provisionally typed the specimens into one of two main categories: 1) epithelial carcinomas of the lung, or 2) other malignancies. Furthermore, epithelial carcinomas were subtyped into nonsmall cell lung cancer (NSCLC), small cell lung cancer (SCLC) or epithelial carcinoma of unsure type. Finally, the cytopathologist was asked to further provisionally subtype NSCLC into one of the following: 1) adenocarcinoma, 2) squamous cell carcinoma, 3) undifferentiated carcinoma, or 4) NSCLC, unsure subtype.

In the case of nonmalignant pathology or malignancies other than epithelial carcinoma, the cytopathologist was asked to give an opinion on the representative nature of the specimen and, if considered representative, which further investigations were appropriate (e.g. performing a cutting needle biopsy or collecting further aspirates for cultures for mycobacteria or fungi).

\section{Cutting needle biopsies}

Cutting needle biopsies were obtained following TTFNA in all cases where the provisional on-site diagnosis was not an epithelial carcinoma of the lung. In cases where the on-site diagnosis was epithelial carcinoma, biopsies were only performed if $\geqslant 2 \mathrm{~cm}$ of safe range could be assured (i.e. no major mediastinal organs, blood vessels and collateral veins were within $2 \mathrm{~cm}$ of the intended CNB path). Superficial veins were avoided. Manually operated 14-gauge Tru-cut biopsy needles with a specimen notch of $20 \mathrm{~mm}$ (Allegiance, Chateaubriand, France) were used. Two or more passes were performed until macroscopically satisfactory material was 
harvested. These specimens were harvested in $4 \%$ formalin and routinely processed for histological evaluation.

\section{Immediate post-procedure care}

The TTFNA and CNB site were re-examined by means of US immediately after the procedures, and a chest radiograph was obtained if the pre- and post-procedure US findings differed and at the discretion of the attending physician. All patients were observed for $\geqslant 2 \mathrm{~h}$ prior to discharge, and complications were noted. The presence or absence of minor or major haemorrhage, as well as iatrogenic pneumothoraces, was specifically documented. Major haemorrhage was defined as any haemorrhage that required additional measures above and beyond localised pressure and superficial sutures.

\section{Further assessment and statistical analysis}

All cytology slides were reviewed in the laboratory by two other cytopathologists, who were blinded to the on-site findings. These cytopathologists had an array of special stains (including immunohistochemistry) at their disposal, and had to concur prior to issuing a final cytological diagnosis. They also had access to the results of the flow cytometry in case of suspected lymphoma. The histological specimens were reviewed by two independent pathologists. Histology was classified as either "diagnostic" or "nondiagnostic" (normal tissue, not representative tissue, or representative but necrotic tissue). Only histological diagnoses or unequivocal cytology (where histology was unavailable and as reported by two laboratory-based cytopathologist) were accepted as diagnostic and used as the gold standard for statistical analyses. Patients in whom the initial procedures failed to yield diagnosis underwent further special investigations in order to obtain a diagnosis. The choice of further invasive investigations was guided by the patient's attending chest physician, and could potentially have included bronchoscopy with direct forceps or transbronchial needle aspiration biopsies (TBNA), CT-guided biopsies, medical thoracoscopy, video-assisted thoracoscopy, and mediastinoscopy or open surgical procedures.

Descriptive statistics and Chi-squared comparisons of proportional data were performed. Unless otherwise stated, data are displayed as mean $\pm \mathrm{SD}$. Standard methods were used to calculate the sensitivity [15].

\begin{tabular}{ll} 
TABLE 1 & $\begin{array}{l}\text { Final established diagnoses of all study subjects } \\
(n=25)\end{array}$ \\
\hline Diagnosis & Subjects \\
\hline Nonsmall cell lung cancer & $11(44)$ \\
Adenocarcinoma & $5(20)$ \\
Squamous cell carcinoma & $1(4)$ \\
Undifferentiated & $5(20)$ \\
Small cell lung cancer & $10(40)$ \\
Lymphoma & $2(8)$ \\
Thymoma & $1(4)$ \\
Mesothelioma & $1(4)$ \\
\hline &
\end{tabular}

\section{RESULTS}

\section{Patients and lesions}

Over the 3-yr period of the present study, a total of 59 consecutive patients with clinical and radiological evidence of SVC obstruction were reviewed by the investigators. Of these, 25 patients $(42.4 \%)$ were included in the study as they remained undiagnosed following the initial special investigations and had an SVC syndrome with an associated mass lesion that abutted or infiltrated the chest wall with an interface of $\geqslant 1 \mathrm{~cm}$. These patients had a mean \pm SD age of $55.8 \pm 11.6 \mathrm{yrs}$, and 16 were male. Fifteen patients had a pulmonary mass lesion, nine patients had a mediastinal mass and only one patient had a pleural-based mass. Of the pulmonary lesions that abutted the chest wall, only four were considered possibly metastatic in nature (all with an associated primary tumour elsewhere in the lungs). The mean depth of lesions (as measured on CT scans, and measured from the point of probable entrance, i.e. measured from the pleural surface, to the most distal tumour limit) was $78 \pm 17 \mathrm{~mm}$. The maximum length of the interface between the lesions and the thoracic wall ranged $10-78 \mathrm{~mm}$, with a mean of $26 \mathrm{~mm}$. Radiological evidence of chest wall invasion was present in nine cases.

Each of the 25 patients enrolled were eventually found to have some form of neoplasm (table 1). Bronchogenic carcinoma was the most common diagnosis, with 11 NSCLC and 10 SCLC diagnosed. The remaining four patients had T-cell lymphoblastic lymphoma, B-cell lymphoma, thymoma and malignant mesothelioma, respectively.

The diagnoses established in the 34 subjects not included in the study population are summarised in table 2 . These diagnoses were established by means of supraclavicular lymph node FNA (diagnostic in four out of six subjects, $67 \%$ ), pleural fluid aspirations (diagnostic in one out of 16,6\%), bronchoscopy with TBNA (diagnostic in 21 out of 25, 84\%), mediastinoscopy (diagnostic in two out of three, 67\%) and open surgical biopsy (diagnostic in one out of one, 100\%). Three patients had clear evidence of SVC thrombosis on contrasted CT scan (all occurred in the setting of intravascular devices) and two remained undiagnosed. Three patients (11\%) who underwent a

\section{TABLE 2 Final established diagnoses of subjects not included in the study $(n=34)$}

\begin{tabular}{lc} 
Diagnosis & Subjects \\
\hline Nonsmall cell lung cancer & $16(47)$ \\
Adenocarcinoma & $7(20)$ \\
Squamous cell carcinoma & $4(12)$ \\
Undifferentiated & $5(15)$ \\
Small cell lung cancer & $9(26)$ \\
Metastatic breast carcinoma & $1(3)$ \\
SvC thrombosis & $3(9)$ \\
Fibrosing mediastinitis & $2(6)$ \\
Immature teratoma & $1(3)$ \\
Undiagnosed & $2(6)$ \\
\hline
\end{tabular}

Data are presented as n (\%). SVC: superior vena cava. 
bronchoscopy experienced minor haemorrhage, while all other procedures were uncomplicated.

\section{US-assisted TTFNA}

US-assisted TTFNAs were performed on all study subjects. Diagnostically useful specimens were obtained from 24 of the 25 study subjects. The on-site cytopathologist identified 21 cases of epithelial carcinoma of the lung and three other malignancies. No ROSE diagnosis was made in one subject. Of the 21 cases of malignant epithelial neoplasm of the lung, 12 were subtyped on ROSE as NSCLC and three as SCLC, whereas six cases could only be subtyped on morphological criteria as unsure type epithelial carcinomas.

After incorporating the results of the immunohistochemistry and flow cytometry (where applicable), two cytopathologists were able to reach a definite diagnosis in 24 out of the 25 cases. It was found that one patient with SCLC was erroneously diagnosed as adenocarcinoma on-site, as both the final cytology and histology confirmed SCLC (fig. 1). The formal laboratory assessment confirmed all other diagnoses, and the 21 cases of epithelial carcinoma of the lung could be subtyped (fig. 1). Furthermore, three cytological diagnoses other than lung cancer could be made in the laboratory: T-cell lymphoma, thymoma and malignant mesothelioma. In all three cases, these diagnoses were confirmed on histology (CNB). The overall diagnostic yield of US-assisted TTFNA was $96 \%$.

\section{CNB}

CNB were performed in all four patients who did not have an on-site diagnosis of epithelial carcinoma and in 12 out of the 21 patients with epithelial carcinoma (57.1\%). Reasons for not performing $\mathrm{CNB}$ included the close proximity of major blood vessels or superficial collateral veins $(n=8)$ and a poor general state $(n=1)$. Diagnostically useful material was obtained in 14 biopsies and the diagnoses made corresponded to the final

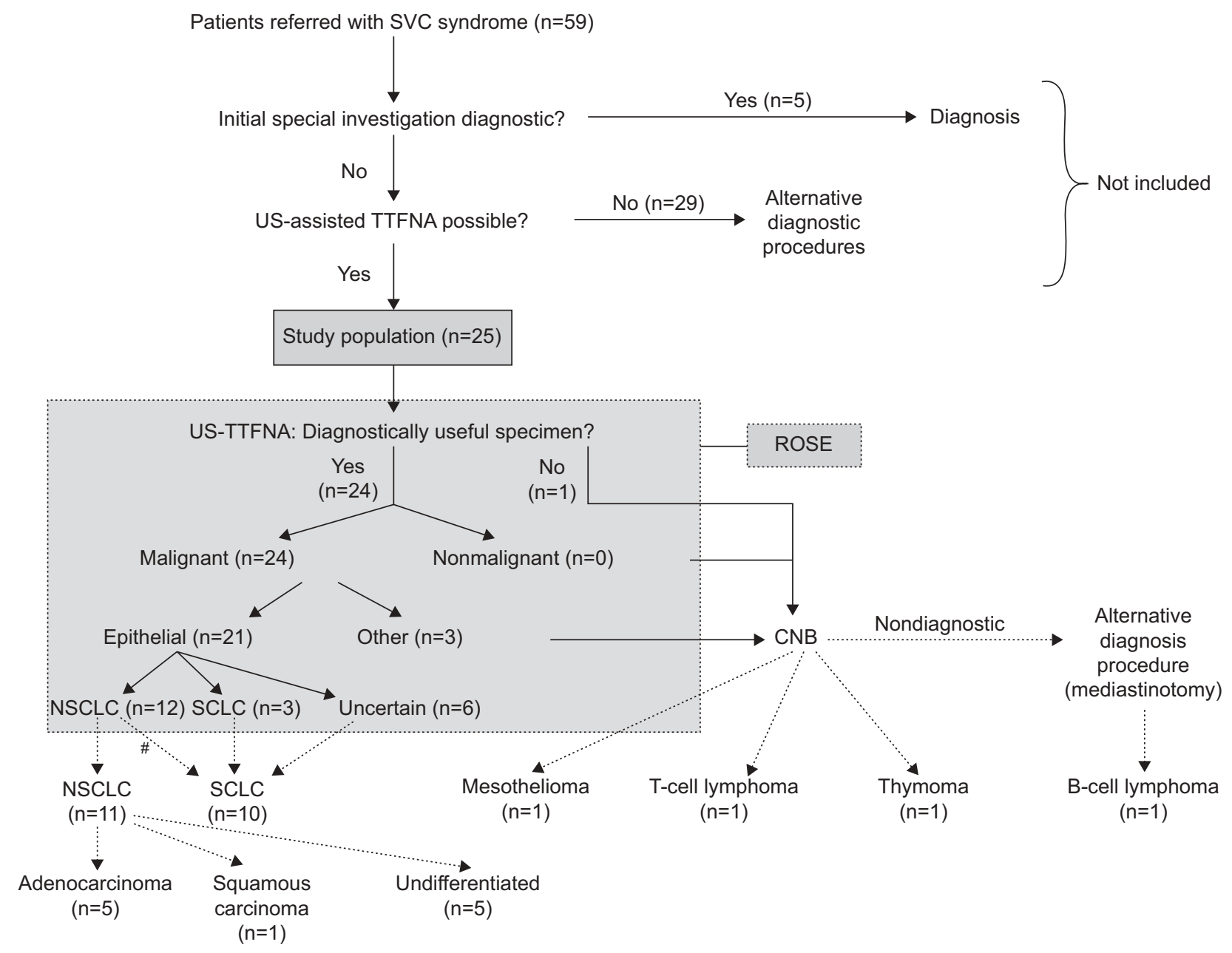

FIGURE 1. The diagnostic pathway followed in the present study. Initial (basic) special investigations included fine needle aspiration (FNA) of supraclavicular lymph nodes and pleural fluid cytology. Of the initial 25 patients, rapid on-site evaluation (ROSE) showed 24 patients to have diagnostically useful specimens obtained by means of ultrasound (US)-assisted transthoracic FNA (TTFNA). Cutting needle biopsies (CNB) were performed in all patients who did not have an on-site diagnosis of epithelial carcinoma of the lung and were deemed safe in 12 out of the 21 patients with epithelial carcinoma. In the case of nonmalignant pathology, further investigations may have included cultures for mycobacteria or fungi (not shown). The final pathological diagnosis was based on histology and unequivocal cytology (reviewed in the laboratory with special stains including immunohistochemistry; see text for detail). SVC: superior vena cava; NSCLC: nonsmall cell lung cancer; SCLC: small cell lung cancer. \#: one case of SCLC was erroneously provisionally diagnosed as NSCLC. 
cytological diagnoses in every case. Nondiagnostic specimens were obtained from two subjects: one patient with undifferentiated NSCLC was convincingly diagnosed on cytology. The other subject, who also escaped diagnosis by TTFNA, was eventually diagnosed as having a B-cell lymphoma by means of an open surgical biopsy (mediastinotomy). The overall diagnostic yield of CNB was $87.5 \%$.

\section{Complications}

The procedures were well tolerated and no pneumothoraces were noted. Only five cases of haemorrhage were documented. One of the 25 patients ( $4 \%$ ) experienced minor haemorrhage following TTFNA, and three out of 16 patients $(18.8 \%)$ following CNB. One patient required a single superficial suture in order to achieve haemostasis. No procedure was complicated by major haemorrhage.

\section{DISCUSSION}

The present prospective study is, to the best of the present authors' knowledge, the largest single-centre study on USassisted TTFNA with ROSE and CNB in the setting of SVC syndrome ever reported. Utilising these minimally invasive techniques, it was possible to accurately diagnose $96 \%$ of all patients who presented with an SVC syndrome with an associated mass lesion that either abutted or infiltrated the chest wall. US-assisted TTFNA with ROSE by a cytopathologist yielded specimens that had a diagnostic yield of $96 \%$. CNB were performed in all cases where a provisional on-site diagnosis of malignant epithelial neoplasm of the lung could not be established, and in $57.1 \%$ of patients with malignant epithelial neoplasm of the lung (biopsies limited due to safety constraints). In this pre-selected group, CNB had a diagnostic yield of $87.5 \%$. A paucity of complications was observed. No pneumothoraces or major haemorrhage was caused, and only mild haemorrhage occurred in $4 \%$ of needle aspirations and $18.8 \%$ of biopsies. These findings are comparable to case series not limited to SVC syndrome [13, 14].

Most experts still utilise cervical mediastinoscopy and anterior mediastinotomy to establish a diagnosis in patients with SVC syndrome secondary to anterior mediastinal mass lesions $[5,6]$. These procedures have a diagnostic yield of almost $100 \%$, but $>15 \%$ of patients suffer a major complication $[5,6]$. Furthermore, patients need to be able to withstand a general anaesthetic and lie in a supine position. The present data suggest that TTFNA and CNB, either US-assisted or CTguided, should be the primary investigation, given the speed, low cost, safety and sensitivity of these minimally invasive techniques. US-assisted TTFNA has many added potential benefits: it can be performed in practically any setting (even outside theatre), on a patient in a variety of positions (including sitting), and a provisional result can be available within minutes if ROSE is utilised [7].

US-assisted biopsy performed by clinicians on peripheral pulmonary and mediastinal mass lesions in the absence of SVC syndrome is a well-established practice [7, 10, 13, 14, 16-24]. SAITO et al. [16] described US-guided mediastinal biopsies $>20$ yrs ago. They were able to diagnose $87 \%$ of all malignant tumours and $67 \%$ of benign masses [16]. YANG et al. [17] showed that US-guided CNB had a diagnostic yield of $94.6 \%$ for subpleural pulmonary tumours, and $88.9 \%$ for mediastinal tumours. The same investigators also pioneered anterior mediastinal biopsies via the supraclavicular approach [18]. Numerous subsequent studies firmly established transthoracic mediastinal FNA and $\mathrm{CNB}$ as investigations with a diagnostic sensitivity ranging $80-90 \%$ [19-22] and as feasible alternatives to mediastinoscopy or diagnostic thoracotomy [23, 24]. The present authors previously reported a combined diagnostic yield for US-assisted TTFNA and CNB of $89 \%$ in 155 consecutive patients with mass lesions that abutted the chest wall [14]. In that study, TTFNA had a significantly higher sensitivity than CNB in diagnosing bronchogenic carcinoma (95 versus $81 \%$; $p=0.006$ ), but $\mathrm{CNB}$ was superior in noncarcinomatous tumours and in benign lesions. SCHUBERT et al. [25] reported similar findings. These observations provide two plausible explanations for the high diagnostic yield observed in the present study. First, $84 \%$ of the present study population had bronchogenic carcinoma and, secondly, biopsies (CNB) were performed in all noncarcinomatous tumours. Decisions concerning the need for biopsies should therefore ideally be guided by the provisional results on ROSE, particularly when CNB are deemed to be risky.

There is a paucity of prospective data on the use of bronchoscopy in the setting of SVC syndrome. SELçUK and FIRAT [26] reported a very high diagnostic yield with TBNA and were able to diagnose $96 \%$ of patients. In fact, only a single case of nonHodgkin's lymphoma escaped diagnosis in their series. Other smaller studies have reported similar findings $[1,27]$. BLACK and ELOUBEIDI [28] were the first to describe the use of oesophageal US-guided FNA specifically in the setting of SVC syndrome.

The findings of the present study support earlier recommendations with regard to the early utilisation of contrasted CT scans in patients who present with suspected SVC obstruction secondary to malignant disease [1, 4, 29-31]. A CT scan can confirm SVC obstruction and the presence of an intrathoracic mass lesion [1, 29-31]. Moreover, in cases where basic investigations, such as sputum cytology, supraclavicular lymph node FNA and pleural aspiration, fail to yield a diagnosis, the findings of a CT scan may guide the choice between further special investigations, chiefly bronchoscopy with TBNA (in the case of more central tumours with mediastinal lymphadenopathy) or US-assisted TTFNA where tumours abut the chest wall [23, 24]. Mediastinoscopy and surgical biopsies should be reserved for patients who remain undiagnosed $[23,24]$.

Thoracic US has limitations; the most relevant and obvious limitation to the present study is its technical inability to visualise mass lesions that do not abut or invade the chest (fig. 2a). Hence, cases not amenable to US-assisted TTFNA were not included in the study (fig. 2b). US-assisted TTFNA was therefore the preferred procedure in only $42.2 \%$ of patients screened, while the majority of the remaining patients were diagnosed by means of bronchoscopy with TBNA.

The present study has limitations. We specifically evaluated the diagnostic yield and safety of US-assisted TTFNA and $\mathrm{CNB}$, and immediately excluded cases that were diagnosed by means of supraclavicular lymph node FNA. High frequency ultrasonography of the supraclavicular fossae might have revealed pathological lymph nodes that escaped palpation [7], 

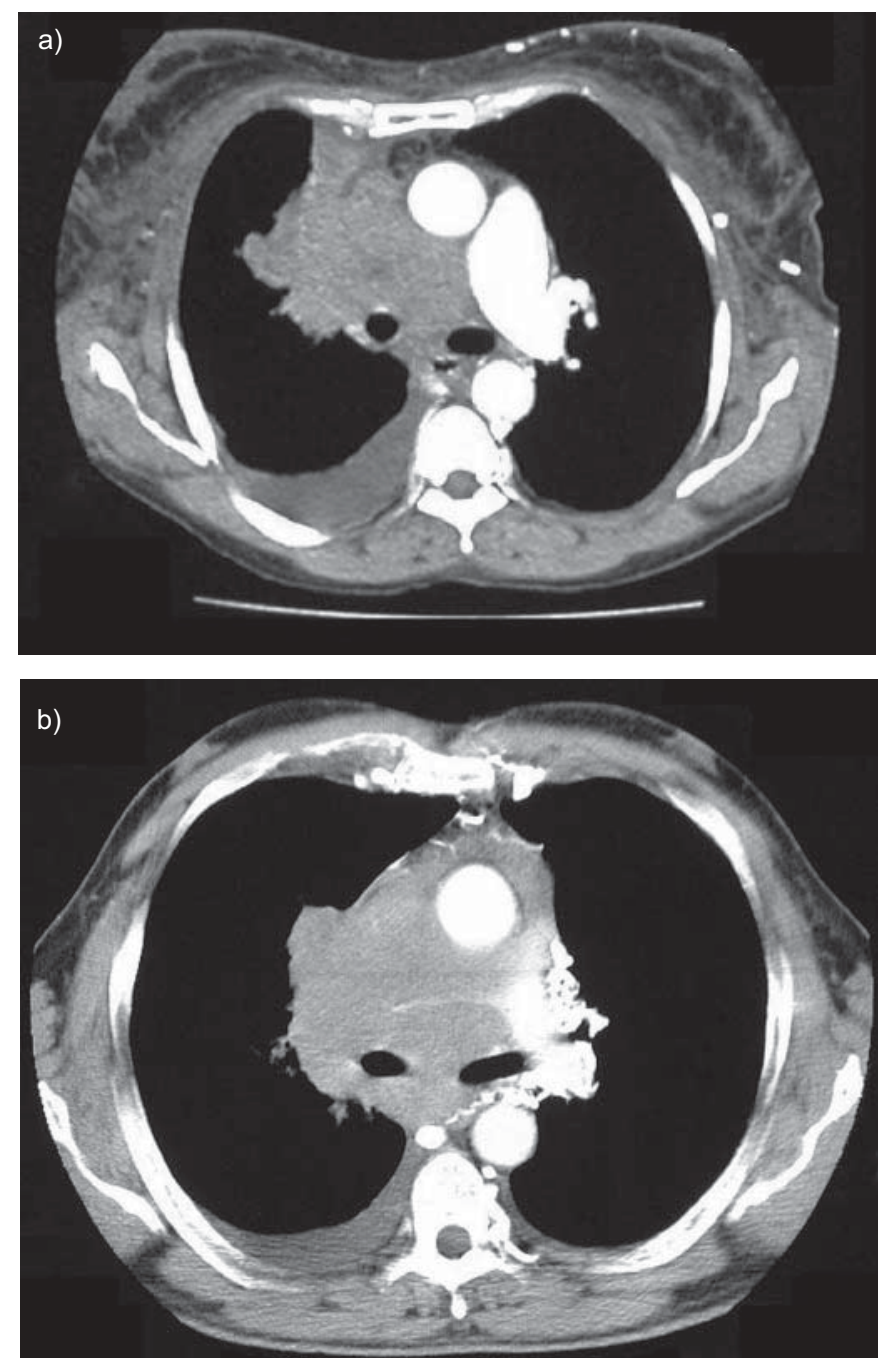

FIGURE 2. Two examples of contrast-enhanced computed tomography (CT) images from patients with superior vena cava (SVC) obstruction that were screened during the present study period. a) The study patient presented with complete SVC obstruction secondary to a tumour that abutted the chest wall, and was included into the study population. b) This CT scan shows a central mass lesion not amenable to ultrasound-assisted transthoracic fine needle aspiration or cutting needle biopsies. Patients with mass lesions not extending to the chest wall were excluded from the present study, and alternative diagnostic techniques were employed in these cases (bronchoscopy with transbronchial needle aspiration in this particular case).

particularly in patients with severe supraclavicular swelling from their SVC syndrome, and in those with radiologically negative nodes on CT scan. This study was not designed to address this issue. Furthermore, we did not compare the diagnostic yield and safety of US-assisted TTFNA and CNB to bronchoscopy in cases amenable to both procedures, nor did we compare clinicians to radiologists with regard to efficiency and healthcare cost.

The single case with an erroneous on-site typing of bronchogenic carcinoma deserved to be highlighted. The diagnosis was revised on the same cytological specimen after full laboratory assessment. More errors in typing may have been made by inexperienced personnel. It should therefore be stressed that the main purpose of ROSE is to ascertain the presence of diagnostically useful material and not to make final diagnoses per se $[14,16]$. Vital decisions on patient management should therefore never be solely based on the ROSE diagnosis.

In conclusion, we were able to show that US-assisted TTFNA with rapid on-site evaluation and CNB performed by a clinician had a high diagnostic yield and were safe in the setting of SVC syndrome with an associated mass lesion that abutted the chest wall. US-assisted TTFNA (with ROSE) may be the initial investigation of choice in suspected bronchogenic carcinoma, whereas both TTFNA and CNB need to be performed in all other cases.

\section{REFERENCES}

1 Wilson LD, Detterbeck FC, Yahalom J. Superior vena cava syndrome with malignant causes. N Engl J Med 2007; 356: 1862-1869.

2 Armstrong BA, Perez CA, Simpson JR, Hederman MA. Role of irradiation in the management of superior vena cava syndrome. Int J Radiat Oncol Biol Phys 1987; 13: 531-539.

3 Parish JM, Marschke RF Jr, Dines DE, et al. Etiologic considerations in superior vena cava syndrome. Mayo Clin Proc 1981; 56: 407-413.

4 Plekker D, Ellis T, Irusen EM, Bolliger CT, Diacon AH. Clinical and radiological grading of superior vena cava obstruction. Respiration 2008; 76: 69-75.

5 Mineo TC, Ambrogi V, Nofroni I, Pistolese C. Mediastinoscopy in superior vena cava obstruction: analysis of 80 consecutive patients. Ann Thorac Surg 1999; 68: 223-226.

6 Dosios T, Theakos N, Chatziantoniou C. Cervical mediastinoscopy and anterior mediastinoscopy in superior vena cava. Chest 2005; 128: 1551-1556.

7 Koegelenberg CFN, Bolliger CT, Diacon AH. Pleural Ultrasound. In: Light RW, Lee YC, eds. Textbook of Pleural Disease. 2nd Edn. London, Hodder \& Stoughton, 2008; pp. 275-283.

8 Beckh S, Bolcskei PL, Lessnau KD. Real-time chest ultrasonography: A comprehensive review for the pulmonologist. Chest 2002; 122: 1759-1773.

9 Tsai TH, Yang PC. Ultrasound in the diagnosis and management of pleural disease. Curr Opin Pulm Med 2003; 9: 282-290.

10 Diacon AH, Theron J, Bolliger CT. Transthoracic ultrasound for the pulmonologist. Curr Opin Pulm Med 2005; 11 307-312.

11 Mayo PH, Doelken P. Pleural ultrasonography. Clin Chest Med 2006; 27: 215-217.

12 Evans AL, Gleeson FV. Radiology in pleural disease: state of the art. Respirology 2004; 9: 300-312.

13 Diacon AH, Schuurmans MM, Theron J, et al. Safety and yield of ultrasound assisted transthoracic biopsy performed by pulmonologists. Respiration 2004; 71: 519-522.

14 Diacon AH, Theron J, Schubert P, et al. Ultrasound-assisted transthoracic biopsy: fine-needle aspiration or cuttingneedle biopsy? Eur Respir J 2007; 29: 357-362. 
15 Fisher L, van Belle G. Biostatistics: a methodology for the health sciences. New York, Wiley, 1993; p. 206.

16 Saito T, Kobayashi H, Sugama Y, et al. Ultrasonically guided needle biopsy in the diagnosis of mediastinal masses. Am Rev Respir Dis 1988; 138: 679-684.

17 Yang PC, Chang DB, Yu CJ, et al. Ultrasound guided core biopsy of thoracic tumors. Am Rev Respir Dis 1992; 146: 763-767.

18 Yang PC, Chang DB, Lee YC, Yu CJ, Kuo SH, Luh KT. Mediastinal malignancy: ultrasound guided biopsy through the supraclavicular approach. Thorax 1992; 47: 377-380.

19 Ikezoe J, Morimoto S, Arisawa J, Takashima S, Kozuka T, Nakahara K. Percutaneous biopsy of thoracic lesions: value of sonography for needle guidance. AJR Am J Roentgenol 1990; 154: 1181-1185.

20 Sawhney S, Jain R, Berry M. Tru-Cut biopsy of mediastinal masses guided by real-time sonography. Clin Radiol 1991; 44: 16-19.

21 Ko JC, Yang PC, Yuan A, et al. Superior vena cava syndrome. Rapid histologic diagnosis by ultrasoundguided transthoracic needle aspiration biopsy. Am J Respir Crit Care Med 1994; 149: 783-787.

22 Samad SA, Sharifah NA, Zulfiqar MA, Maimunah A, Yahya A, Zainudin W. Ultrasound guided percutaneous biopsies of suspected mediastinal lesions. Med J Malaysia 1993; 48: 421-426.
23 Tikkakoski T, Lohela $\mathrm{P}$, Leppänen M, Apaja-Sarkkinen M, Typpäo T, Mäkäräinen $\mathrm{H}$. Ultrasound-guided aspiration biopsy of anterior mediastinal masses. J Clin Ultrasound 1991; 19: 209-214.

24 Andersson T, Lindgren PG, Elvin A. Ultrasound guided tumour biopsy in the anterior mediastinum. An alternative to thoracotomy and mediastinoscopy. Acta Radiol 1992; 33: 423-426.

25 Schubert P, Wright AC, Louw M, et al. Ultrasound-assisted transthoracic biopsy: cells or section? Diagn Cytopathol 2005; 33: 233-237.

26 Selçuk ZT, Firat P. The diagnostic yield of transbronchial needle aspiration in superior vena cava syndrome. Lung Cancer 2003; 42: 183-188.

27 Witt C, Schmidt B, Borges AC, Doerffel W, Baumann G, Romaniuk P. Superior vena cava syndrome. From the bronchus to the vessel. Diagn Ther Endosc 1997; 4: 83-93.

28 Black KR, Eloubeidi MA. Superior vena cava syndrome: diagnosis of etiology with EUS-guided FNA. Gastrointest Endosc 2008; 67: 568-570.

29 Abner A. Approach to the patient who presents with superior vena cava obstruction. Chest 1993; 103: 394S-397S.

30 Raptopoulos V. Computed tomography of the superior vena cava. Crit Rev Diagn Imaging 1986; 25: 373-429.

31 Yedlicka JW, Schultz K, Moncada R, Flisak M. CT findings in superior vena cava obstruction. Semin Roentgenol 1989; 24: 84-90. 